\title{
Optimized merging of search coil and fluxgate data for MMS
}

\author{
David Fischer ${ }^{1,5}$, Werner Magnes ${ }^{1}$, Christian Hagen ${ }^{1}$, Ivan Dors ${ }^{2}$, Mark W. Chutter ${ }^{2}$, Jerry Needell ${ }^{2}$, Roy B. Torbert ${ }^{2}$, \\ Olivier Le Contel ${ }^{3}$, Robert J. Strangeway ${ }^{4}$, Gernot Kubin ${ }^{5}$, Aris Valavanoglou ${ }^{1}$, Ferdinand Plaschke ${ }^{1}$, \\ Rumi Nakamura $^{1}$, Laurent Mirioni ${ }^{3}$, Christopher T. Russell ${ }^{4}$, Hannes K. Leinweber ${ }^{4}$, Kenneth R. Bromund ${ }^{6}$, \\ Guan Le $^{6}$, Lawrence Kepko ${ }^{6}$, Brian J. Anderson ${ }^{7}$, James A. Slavin ${ }^{8}$, and Wolfgang Baumjohann ${ }^{1}$ \\ ${ }^{1}$ Space Research Institute, Austrian Academy of Sciences, Graz, Austria \\ ${ }^{2}$ Space Plasma/Magnetospheric Physics, University of New Hampshire, Durham, USA \\ ${ }^{3}$ Laboratoire de Physique des Plasmas - UMR7648, CNRS/École Polytechnique/UPMC/Univ. Paris Sud/Obs. de Paris, \\ Paris, France \\ ${ }^{4}$ Institute of Geophysics and Planetary Physics, University of California, Los Angeles, USA \\ ${ }^{5}$ Signal Processing and Speech Communication Laboratory, Graz University of Technology, Graz, Austria \\ ${ }^{6}$ Goddard Space Flight Center, NASA, Greenbelt, USA \\ ${ }^{7}$ Applied Physics Laboratory, John Hopkins University, Laurel, USA \\ ${ }^{8}$ Climate and Space Sciences and Engineering, University of Michigan, Ann Arbor, USA
}

Correspondence to: David Fischer (david.fischer@oeaw.ac.at)

Received: 14 April 2016 - Published in Geosci. Instrum. Method. Data Syst. Discuss.: 6 June 2016

Revised: 19 September 2016 - Accepted: 30 September 2016 - Published: 17 November 2016

\begin{abstract}
The Magnetospheric Multiscale mission (MMS) targets the characterization of fine-scale current structures in the Earth's tail and magnetopause. The high speed of these structures, when traversing one of the MMS spacecraft, creates magnetic field signatures that cross the sensitive frequency bands of both search coil and fluxgate magnetometers. Higher data quality for analysis of these events can be achieved by combining data from both instrument types and using the frequency bands with best sensitivity and signalto-noise ratio from both sensors. This can be achieved by a model-based frequency compensation approach which requires the precise knowledge of instrument gain and phase properties. We discuss relevant aspects of the instrument design and the ground calibration activities, describe the model development and explain the application on in-flight data. Finally, we show the precision of this method by comparison of in-flight data. It confirms unity gain and a time difference of less than $100 \mu$ s between the different magnetometer instruments.
\end{abstract}

\section{Introduction}

The MMS mission (Magnetospheric Multiscale; Burch et al., 2015) is comprised of four satellites that are used to measure plasma processes in the Earth's magnetosphere. The main mission target is the exploration of magnetic reconnection. To support measurements down to electron scales, the satellites are flying in a tight formation with distances down to $10 \mathrm{~km}$. One of the main measurement quantities used to characterize plasma processes is the magnetic field. It is measured by three instruments which are part of the MMS FIELDS suite (Torbert et al., 2014): the analog fluxgate magnetometer (AFG), the digital fluxgate magnetometer (DFG) and the search coil magnetometer (SCM). As the measured plasma structures can have speeds from 10 to $1000 \mathrm{~km} \mathrm{~s}^{-1}$, high measurement accuracy in both magnetic field magnitude and timing is required over a wide frequency range, e.g., to calculate speed and direction of a front crossing the tetrahedron satellite configuration.

The two fluxgate magnetometers are able to measure the magnetic field between DC and $64 \mathrm{~Hz}$. The noise floor is around $5 \mathrm{pT} \mathrm{Hz}^{-1 / 2}$ at $1 \mathrm{~Hz}$ and it increases towards lower frequencies (Russell et al., 2014). The frequency responses of both fluxgate magnetometers are flat for lower frequen- 
cies and correspond to a low pass with low filter order and a corner frequency around $30 \mathrm{~Hz}$. Therefore, fluxgate data are typically used for scientific analysis without any compensation of the frequency-dependent gain and phase characteristics.

The search coil magnetometer is suitable for measuring much higher frequencies, but its sensitivity at lower frequencies is limited by the underlying induction principle. Its frequency response represents a transfer function of higher order and requires compensation based on ground calibration measurements (Le Contel et al., 2014).

A noise floor comparison, which partly reflects the frequency response, is shown in Fig. 1. The plot compares the digital output of the DFG with the analog output from the SCM. The frequency range of equal noise floor is between 3 and $6 \mathrm{~Hz}$, although this may vary across different instrument implementations. With the wide frequency range of plasma events mentioned above, it is desirable to have a common merged product that combines the best data of both instruments, i.e., all available frequency bands with lowest possible noise floor. This is particularly useful for observations of electron diffusion regions and thin current sheets which feature signatures in the frequency range from 0.5 to $20 \mathrm{~Hz}$ (Torbert et al., 2014).

A similar approach was already used on magnetic field data from the Cluster and Themis missions, but in both cases it was based on limited on-ground frequency response calibration and usage of in-flight data for calibration. The SCM frequency characteristics were in principle known (Cornilleau-Wehrlin et al., 1997; Roux et al., 2008), but the knowledge of absolute time was limited. The knowledge of the frequency response of the fluxgates (Balogh et al., 1997; Auster et al., 2008) was limited to discrete frequency points in amplitude and low accuracy on end-to-end phase and timing information. Merging for data analysis was therefore only possible using this limited data and in-flight comparison (Alexandrova et al., 2004). Furthermore, comparative calibration of the SCMs was done by matching to fluxgate data at very low frequencies, which are not influenced by the fluxgate low pass characteristic (Robert et al., 2014).

Only the precise knowledge of the magnitude and phase response of both instrument types allows for accurate merging of the respective data, keeping intact phase and gain relations between different frequency components of the observed magnetic signatures. Therefore the shape of the signatures is preserved in the merged data product, as higher frequency components of steeper slopes stay phase aligned. Hence, the merged data are well suited for the analysis of internal fine structures of magnetic signatures as well as for high precision determinations of dipolarization front speed and direction by multi-spacecraft timing analysis. In addition, the comparative calibration of gain and alignment between SCM and AFG/DFG is massively improved in flight. Without the precise knowledge of their frequency responses either data with low signal-to-noise ratio (SNR) from the

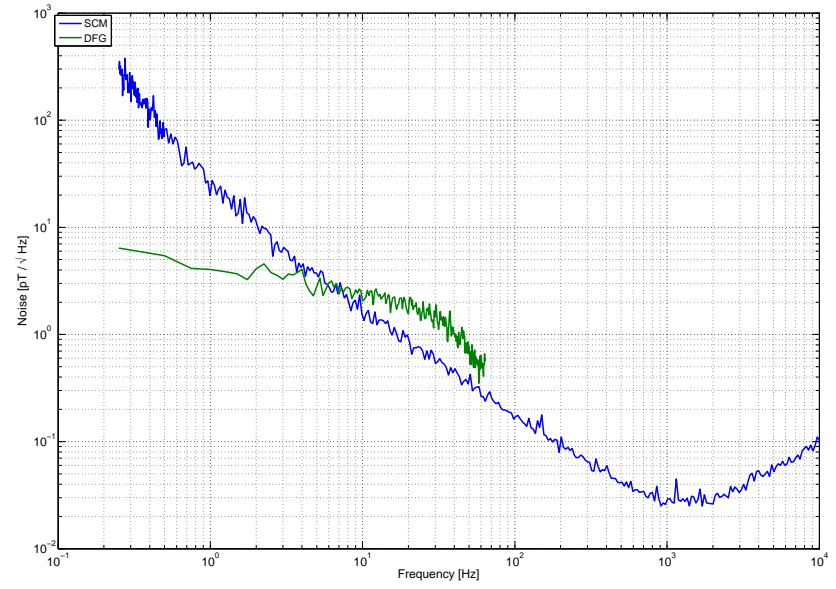

Figure 1. Comparison of search coil (SCM) and fluxgate (DFG) noise floor.

SCM (i.e., at the satellite spin frequency) or data with little phase knowledge from the fluxgate magnetometers have to be used for comparative calibration. This results in alignment errors and inaccurate gain factors. With this knowledge, data from the complete common frequency band can be used and areas with good SNR can be selected.

The MMS FIELDS team invested significant efforts in instrument design and test to ensure that end-to-end timing and frequency response information is already available from onground calibration. Design efforts include a common master clock and synchronization, which enables sampling of SCM, AFG and DFG with constant phase relation, as well as high precision time stamping. The target of these efforts was to reach an absolute time tagging accuracy of $100 \mu$ s across the full bandwidth despite the heterogeneous architecture of the instruments. This required common clocks and synchronization, as instruments are sampled by different means. AFG and DFG are sampled by their own electronics, whereas the SCM is sampled by the digital signal processing unit (DSP; Ergun et al., 2016). In both cases, depending on downlink data rate, digital filters for sample rate conversion are also applied, which also needs to be taken into account for time tagging.

This work deals with the method to create a merged $1024 \mathrm{~Hz}$ data product from $128 \mathrm{~Hz}$ fluxgate and $8192 \mathrm{~Hz}$ search coil data. In the first step this required the verification of absolute timing and the identification of the instrument's frequency responses in an end-to-end test on the ground. In the next step data from these tests have been used to create instrument models, which can be employed for inverting the instrument's frequency responses and to make the data fit for precise merging. In the final part, in-flight data were merged using these models and the results were evaluated to verify the timing precision of the complete process. 


\section{Approach}

Characterization of an unknown system is done by using known input signals, measuring the system response and creating a model using both signals. For magnetometers the input signal has to be a magnetic field stimulus and the system response is the digital measurement result. Since both instrument types can be considered as linear and time-invariant for the case of a merged data product, it is possible to describe them with a model based on finite (FIR) or infinite impulse response filters (IIR). A suitable magnetic field stimulus can, for example, be created by a solenoid coil system. Amplitude and phase of the magnetic field are linked to the applied current.

\subsection{On-ground calibration and measurement setup}

Figure 2 shows a block diagram of the setup used for the frequency response calibration. All magnetic field sensors of the FIELDS instrument suite were placed in a mu-metal can and the stimulus signal was applied using a current generator and a solenoid coil. AFG and DFG were digitized by the respective front-end electronics, while SCM signals first passed through a preamplifier and were then sampled by the DSP unit. The data streams were then processed by the FIELDS central data processing unit (CDPU).

The current generator and the CDPU were synchronized by a common $1 \mathrm{~Hz}$ time reference clock. All data recorded within FIELDS were time stamped relative to this reference. Furthermore all instruments within FIELDS were synchronized using common master and synchronization clocks. A more detailed description of this mechanism is available in the FIELDS instrument paper (Torbert et al., 2014).

The current generator that was constructed for this test is able to drive an arbitrary waveform current. It is generated by an internal digital signal generator. This signal generator is operated at a sampling frequency of $4096 \mathrm{~Hz}$, which is synchronized to the reference. This sampling clock is derived from a $67 \mathrm{MHz}$ master clock internal to the current generator, but instead of using a fixed divider, additional clock cycles are added (or subtracted) as required to keep it synchronous to the $1 \mathrm{~Hz}$ time reference. These added clock cycles must be considered as artificial jitter, which in principle creates additional noise in the stimulus signal. The maximum amplitude of this noise can be calculated by using the maximum possible signal change within the maximum jitter time. For a jitter of $15 \mathrm{~ns}$ and a maximum signal frequency of $1024 \mathrm{~Hz}$ this results in an SNR of approximately $80 \mathrm{~dB}$, which was sufficiently low for this purpose. As every real current source will produce small differences between desired and achieved current, an additional current measurement was mandatory. A comparison of the generated current and the $1 \mathrm{~Hz}$ time reference signals shows a time deviation in the sub-microsecond range.

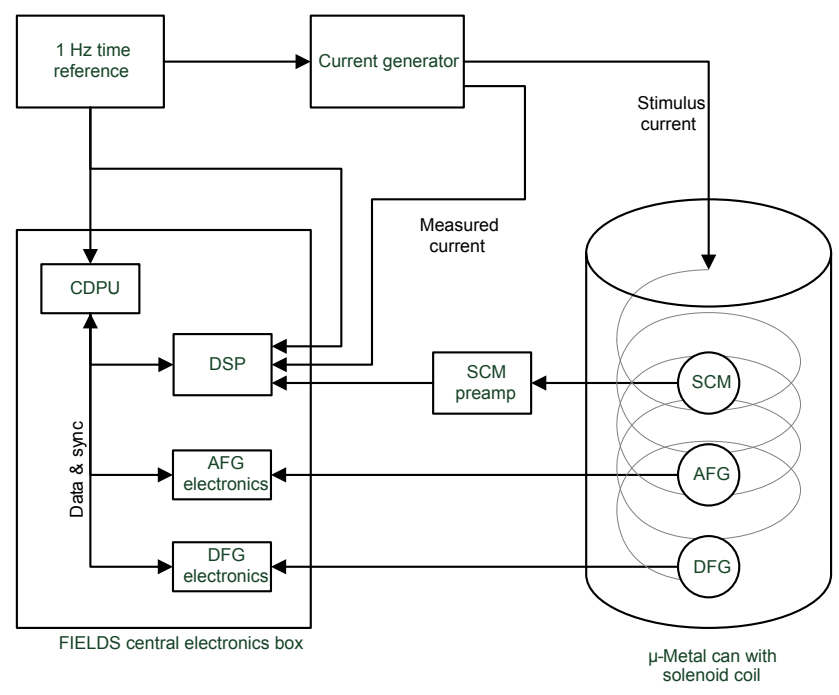

Figure 2. Test setup for instrument frequency response measurements.

The current stimulus was driven through a solenoid coil in a mu-metal shielding, which attenuates external fields during the measurement. The influence of this coil/can system is minimal in the frequency range of interest from DC to $512 \mathrm{~Hz}$; therefore the generated field could still be considered as proportional to the measured current.

The stimulus waveforms used for testing need to cover the whole frequency range of interest. Commonly used test signals for identifying unknown frequency responses are discrete frequency sines (using interpolation in between), sine sweeps and noise signals. Discrete and sweep sine signals have the advantage that the signal frequency for any given time is known and therefore all signals with different frequencies can be removed by bandpass filtering. However, longer measurement times are needed to excite all frequencies. Noise measurements excite all frequencies within a short period of time, but this method is sensitive to all additional noise sources within the analyzed frequency band. However, any uncorrelated noise can be reduced by longer measurements and averaging.

For the FIELDS frequency response calibration three different tests were conducted with each of the instrument suites of the four MMS satellites. In a first test, the $1 \mathrm{~Hz}$ time reference was connected to one of the additional voltage channels of the DSP. In a second test, the instrument responses were measured only at a few discrete frequencies with sine stimuli. In a third test, pink noise with a bandwidth from DC to $1024 \mathrm{~Hz}$ and a duration of $5 \mathrm{~min}$ was applied to all sensors and the instrument response was recorded. The choice of pink over white noise was driven by the sensitivity curve of the SCM, as the higher frequency components of a white signal would drive the SCM to saturation even when the measurement output at lower frequencies is small. 


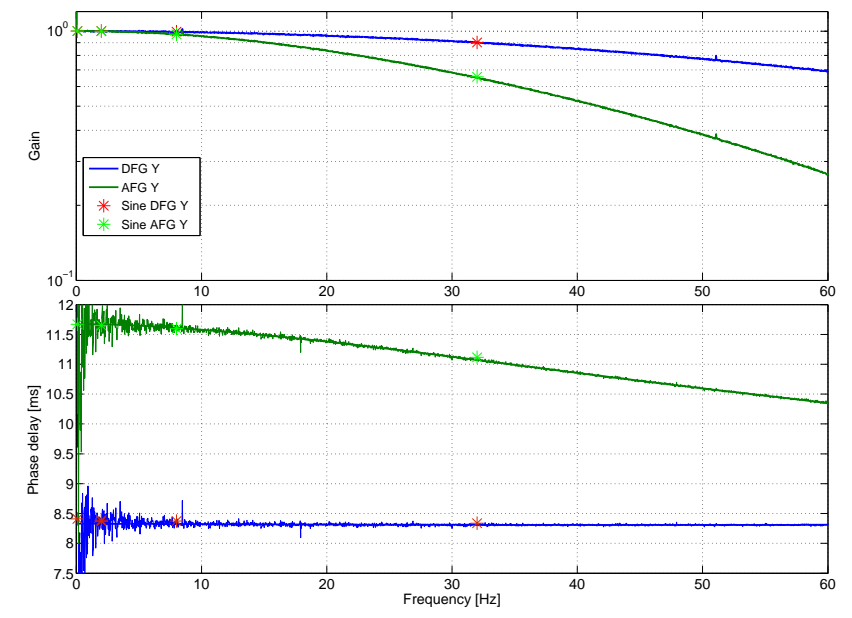

Figure 3. Frequency response of AFG and DFG measured with FFT estimation method and sine signals. FFT length is 4096; overlap is 1023; rectangular window; data duration of $5 \mathrm{~min}$.

In addition to the internal measurement of the current generator, a voltage fully representative to the current stimulus was connected to a DSP voltage channel. This way, the current was also recorded synchronously to the FIELDS clock and so it could be used in later stages without the need for further resampling or time shifting. Additionally, this resulted in faster data transfer and easier data formatting.

\subsection{Results of on-ground calibration}

Using data from the first test, which was the digitizing of the time reference signals, it was possible to measure the delay introduced in sampling and time stamping of the DSP voltage channels by comparing the sampled waveform of the reference clock and its time stamp. As the $1 \mathrm{~Hz}$ time reference is giving the reference for the beginning of a full second, the clock edge within the sampled waveform should ideally be time stamped with this second. The measured deviation from the expected delay was less than $7 \mu \mathrm{s}$.

The results of the second test based on the sine signals delivered a highly accurate measurement of gain and phase at discrete frequencies and an initial approximation of the frequency response, for both the magnetic field instruments and the current measurement via the DSP voltage channel.

These first two tests showed that the DSP voltage channels had sufficiently flat frequency response and that the knowledge on timing accuracy was better than $1 \mu \mathrm{s}$. The later noise tests were therefore conducted using the current measurements of the DSP channels, as this resulted in reduced effort in calculations. The additional delay of these channels was accounted for in all further tests. For verification purposes, both the sine and time reference measurements were included in all further test series to ensure that no changes occurred in the setup.
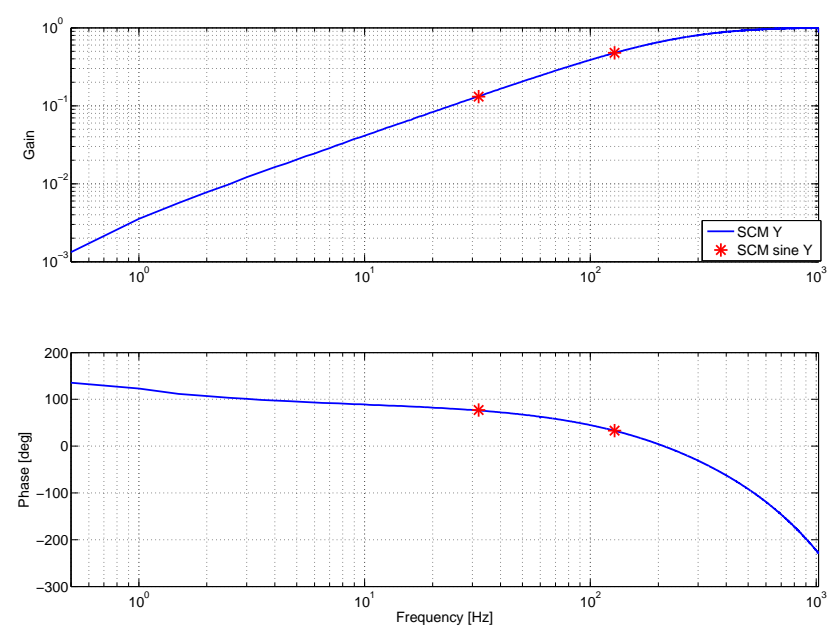

Figure 4. Frequency response of SCM measured with FFT estimation method and sine signals. FFT length is 32768 ; overlap is 32767 ; rectangular window; data duration of $5 \mathrm{~min}$.

The resulting data of the third test with applied noise signals were used to create an initial estimate of the full instrument frequency responses. This estimate can be found by dividing the fast Fourier transform (FFT) of stimulus and instrument measurements, which is the frequency domain equivalent of deconvolution.

Unfortunately, this method is not necessarily delivering an estimate that is fully representative for the system. The FFT method implies that a single transform window only contains the convolution of the transfer function and a stimulus. In reality the beginning of each FFT window contains a part of the response to previous stimulus data and a part of the response to the current stimulus is truncated. In addition, noise and distortion within the window are also considered as part of the frequency response. The problem of previous as well as truncated response data can be minimized by choosing a sufficiently large FFT length and by using windowing, overlapping and averaging. The problem of measurement noise is more complicated. Although normal noise can be reduced by averaging, this is not the case for systematic distortion like powerline tones $(50 / 60 \mathrm{~Hz}$ and harmonics). In this case the resulting estimate would have a changed frequency response at the respective frequencies. The FFT-based estimate can therefore not be used as a model.

Figures 3 and 4 show examples of both sine- and noisebased frequency response estimate results from the $y$ axis of flight model 4 (used on MMS 3). Figure 3 shows that AFG and DFG have the expected low pass characteristics. The DFG has a higher corner frequency and constant phase delay. The noise visible in the phase delay plot results from the translation of phase noise to phase delay, which increases the noise at lower frequencies. Found delays for DFG match the expected digital delay with a maximum deviation of $30 \mu \mathrm{s}$. The delay and gain curves for other axes of the AFG have a 
slightly different characteristic due to the differences in analog elements.

Figure 4 shows the lower end of the expected SCM bandpass characteristic, which also matches the reference measurements taken by the SCM team in Chambon-la-Forêt (Le Contel et al., 2014). The frequency response for higher frequencies was not measured, as this was not required for the merged $1024 \mathrm{~Hz}$ data product.

The absolute timing of the FFT estimates was calculated by adding the delays of the DSP voltage channels known from the first two tests. A comparison between FFT estimate with added delay and the sine measurements, which do not include the current measurement via the DSP channel, shows good agreement and is therefore verifying the correct handling of delays in the DSP voltage channels.

\subsection{Model development}

The SCM model was chosen according to a theoretical model from the SCM team (Le Contel et al., 2014, Appendix A). As parts of the frequency transfer function of this model are far above the frequency band of interest, the model was reduced to a second-order IIR filter-based model and a fractional sample Lagrange delay. Equation (1) shows the continuous time representation of the filter, which was converted to discrete time using the bilinear transform.

$h(s)=\frac{G\left(\frac{1}{\omega_{1}} s\right)\left(\frac{1}{\omega_{2}} s\right)}{\left(\frac{1}{\omega_{1}} s+1\right)\left(\frac{1}{\omega_{2}} s+1\right)}$

The IIR model was then optimized to fit the measurement results in frequency domain, using a complex least mean square error fit (Eq. 2).

$\omega_{1,2}=\arg \min E\left\{\left(H_{\mathrm{FFT}}(f)-F T\left(h\left(z, \omega_{1,2}\right)\right)\right)^{2}\right\}$

Inversion of this optimized model is not directly possible, as it is not minimum phase (Oppenheim et al., 1999) - which means that the inverted model is unstable and has poles outside of the unit circle. This is also visible by the differentiating feature of the SCM. If this feature would be inverted, the resulting integrator would produce infinite values even with small DC inputs. As the lower parts of the spectrum are not used in the later merged product, it is possible to design a similar IIR transfer function with nonzero DC gain and minimum phase property by just adding a small additional coefficient, thus changing the original numerator polynomial to have all poles within the unit circle. The resulting inverse model is a low shelving filter with a DC gain of $220 \mathrm{~dB}$, which is close to the unmodified model for all frequencies above $0.1 \mathrm{~Hz}$.

The main contributor to the frequency response of the DFG is the digital averaging filter used for the DFG internal downsampling (Magnes et al., 2003). Also, this filter cannot be considered as minimum phase and does not allow direct
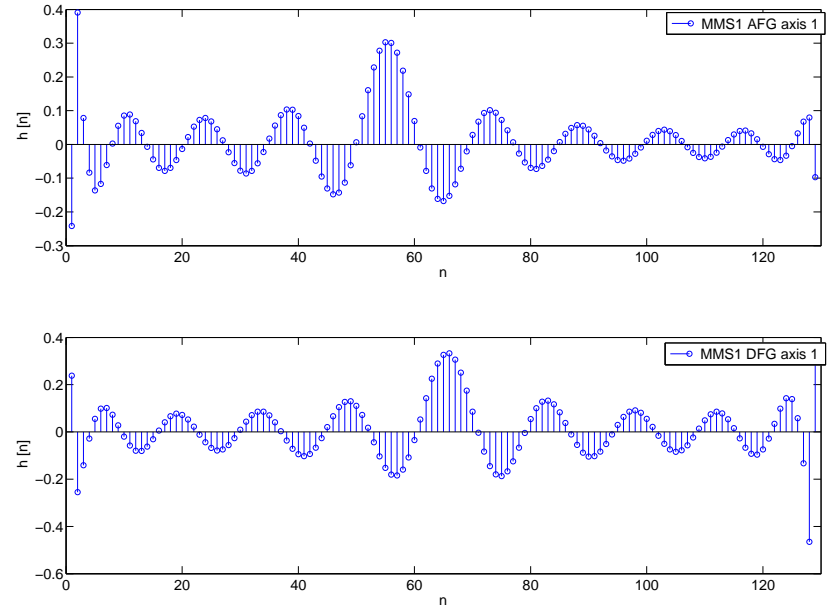

Figure 5. Exemplary impulse response of fluxgate compensation filter model.

inversion. However, since only the spectral part below $64 \mathrm{~Hz}$ is used in the final $1024 \mathrm{~Hz}$ product, the properties of an inversion filter are irrelevant above these frequencies. This fact leaves some degrees of freedom for an optimization solution. The basic model in this case is a 128th-order FIR model that was derived using a Wiener-Hopf solution (Haykin, 2002). This method delivers a model that converts the input stimulus to a model output signal which tracks the real instrument measurements with minimum mean square error.

For the DFG this principle was inverted by exchanging input and output, thus creating an inverse model that can directly be applied on instrument data to reconstruct the "original" magnetic field data. Additionally, the instrument measurement data are compensated for the delay before modeling, as otherwise the model would have to resemble both delay and frequency response of the instrument. Keeping these delays within the model would in principle only cause a shift of the model coefficients by adding zero coefficients at the beginning. This addition would increase the filter order that is used for optimization and instead of keeping these coefficients at zero, an optimal solution would try to model just the instrument noise to get to a minimum error. Introducing a time shift is therefore reducing model order and is avoiding coefficients that just model the noise.

The same approach, although with different delay compensation, was used on the AFG data. In this case the characteristic part of the filter is the analog low pass of the feedback regulation loop, which also introduces non-constant group delay. The respective modeling process of each instrument was applied individually to each of the instrument axes. An exemplary impulse response of the compensation filter model for AFG and DFG is presented in Fig. 5.

The found instrument models were transformed to the frequency domain, inverted and compared to the FFT and sinebased frequency responses. Figures 6 to 8 show the differ- 

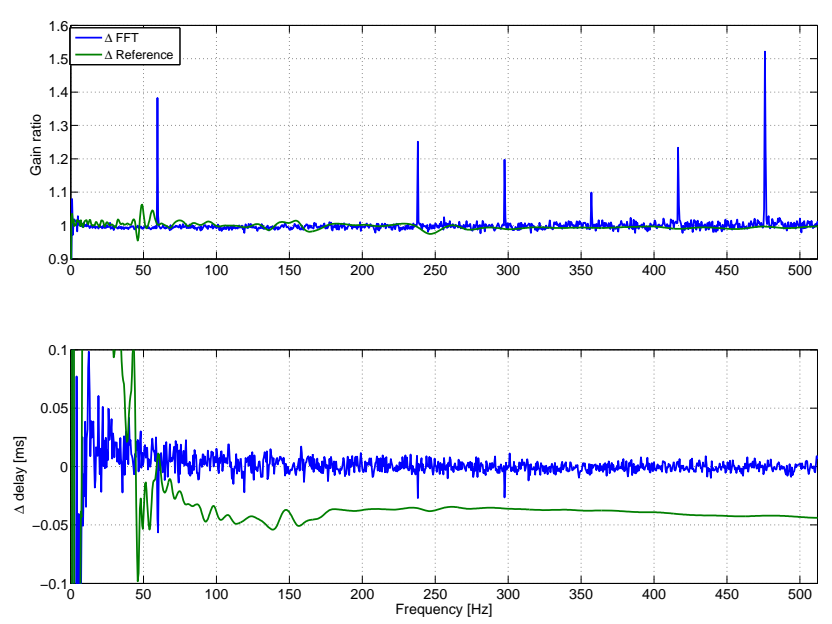

Figure 6. Gain ratio and delay difference between SCM frequency response model and FFT estimate as well as analog reference calibration.
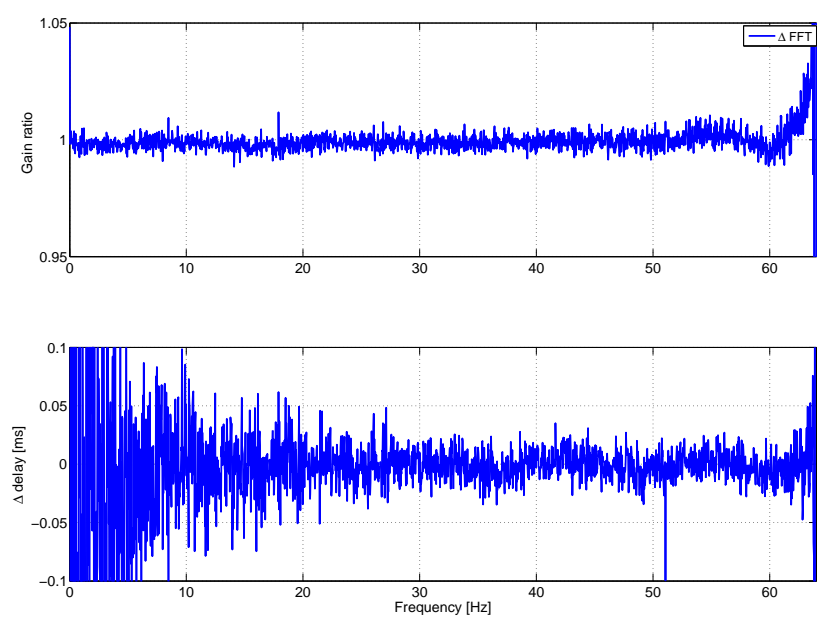

Figure 7. Gain ratio and delay difference between AFG frequency response model and FFT.

ences between FFT-based results, model response and reference calibration (Le Contel et al., 2014). The SCM model comparison in Fig. 6 shows the presence of powerline noise as peaks in amplitude and phase, both in the FFT $(60 \mathrm{~Hz})$ and the reference $(50 \mathrm{~Hz})$ measurements. These peaks are absent in the models and are therefore visible as differences in the comparison. The constant delay between reference and FFT as well as model measurements is due to the fact that the SCM reference calibration was only done using the analog sensor output and does not include all digital delays introduced by sampling.

Data from DFG and AFG presented in Figs. 7 and 8 show a very good match between model and FFT-based frequency response measurement results and, for DFG, also with the theoretical values of the digital averaging filter. The remaining delay variation of around $20 \mu \mathrm{s}$ is well below the $100 \mu \mathrm{s}$
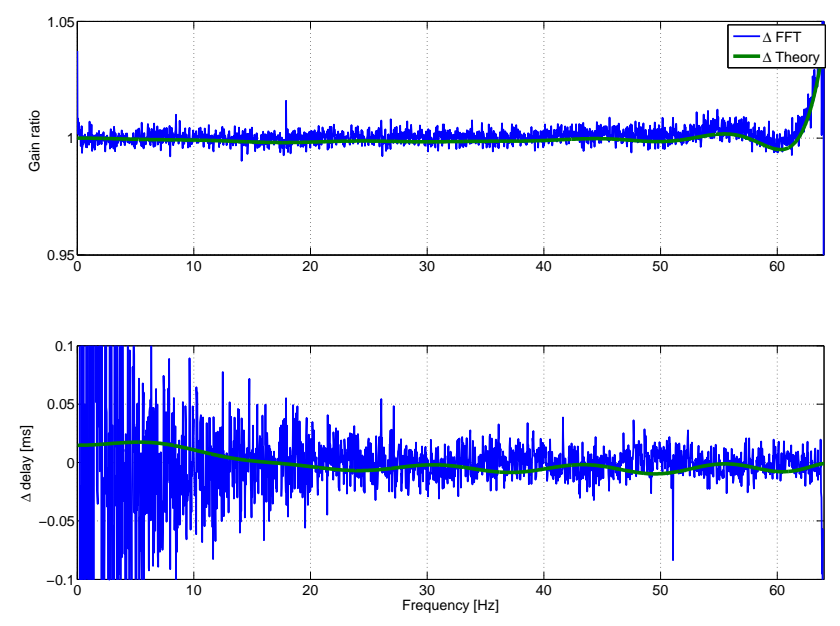

Figure 8. Gain ratio and delay difference between DFG frequency response model and FFT estimate as well as theoretical digital filter response.

goal. It is caused by the limited length FIR implementation, which could be corrected by a filter of higher order - but this has disadvantages, as shown in the next paragraphs.

The last step of model creation was to normalize the models to the results of regular on-ground gain calibration of the instrument teams. For the fluxgates this was done by setting the DC gain to one, so DC calibration would be unchanged. For the SCM it was done by matching the gains of model and on-ground reference calibration around $1 \mathrm{kHz}$.

The filter-based instrument models require an initial filter settling time that is dependent on the filter impulse response length. Data from this period need to be removed from the final data product and therefore produce a data gap. This is of special concern for MMS high sampling rate data, as only short data bursts are transmitted and a loss of many data points cannot be tolerated. Some of this could be mitigated by prefilling with data from lower sampling frequencies, but this process is complex, as multiple frequency response compensation is involved. The best solution is therefore the use of limited length filter functions.

This is already the case for fluxgate model filters, but the IIR characteristic of the SCM requires in principle one more iteration. For later merging, the compensated SCM data are filtered with a high pass filter. These two filter operations for compensation and merging can be combined to a single filter by convolution. The impulse response of this convolved filter is theoretically infinite but practically decaying very fast; e.g., using a 1025 point merging filter (see below) the impulse response decreases to $10^{-13}$ after roughly 1000 points. Numbers of this size are far below the instrument noise and can therefore be neglected. It is therefore possible to replace the IIR filter by its truncated impulse response without relevant changes in the frequency response. For this paper, the 
IIR filter was used as SCM model. A replacement by an FIR model is planned in the future.

Data from both instruments are merged with a crossover filter that is weighting different spectral parts of the instruments according to their properties. An optimum crossover filter set would weigh the different frequency components of the signal based on a comparison of instrument sensitivity, noise floor and possible distortion. These weights can be considered as filter function with variations in frequency domain. A transform of a filter function with high variability will result in a high-order filter function, which will again cause data loss due to filter settling. We therefore chose to implement a windowed FIR low and high pass filter based on the sinc function, which is simply using DFG/AFG data up to $4 \mathrm{~Hz}$ and SCM data above. The sinc function also provides the advantage that design of the respective complementary filter is a simple subtraction from the Dirac delta. This means the sum of the two filters is unity gain. Furthermore, comparison to unfiltered signals is simple due to its constant group delay property.

Here the window length also has to be matched to an acceptable data loss due to filter settling. With larger window size stopband attenuation increases, passband ripple decreases and the crossover characteristic has a steeper slope. Figure 9 shows an example of two filter sets with different window lengths and the resulting differences in attenuation and crossover slope. For initial merging and comparison the 16385 point filter was used, but in later mission phases with shorter data bursts this will be changed to 2049 points or less, depending on available burst data length.

\section{Application}

The final process of merging, which is suitable for automated application, is shown in the data flow diagram in Fig. 10. Apart from the already discussed crossover filters and modelbased frequency response compensation, a few more blocks are present in this diagram. The uncalibrated data files from all instruments (called L1A data according to MMS definition) are passed through a block that handles fragmentation, as data files can have gaps and contiguous data can be distributed over several files.

The resampling block includes antialiasing filters and converts the different data products to the final product rate of $1024 \mathrm{~Hz}$. The first remaining sample in the decimated data product of SCM is selected by looking for the closest neighbor in the fluxgate data, thus minimizing the time distance between those samples. This reduces the amount of time shift needed to synchronize the data to a common sample time basis.

The blocks for timestamp updates and fractional delays are two separate parts of a common mechanism. Fractional delays (less than a sampling period) are required to align data to a common time basis and to compensate absolute time de-

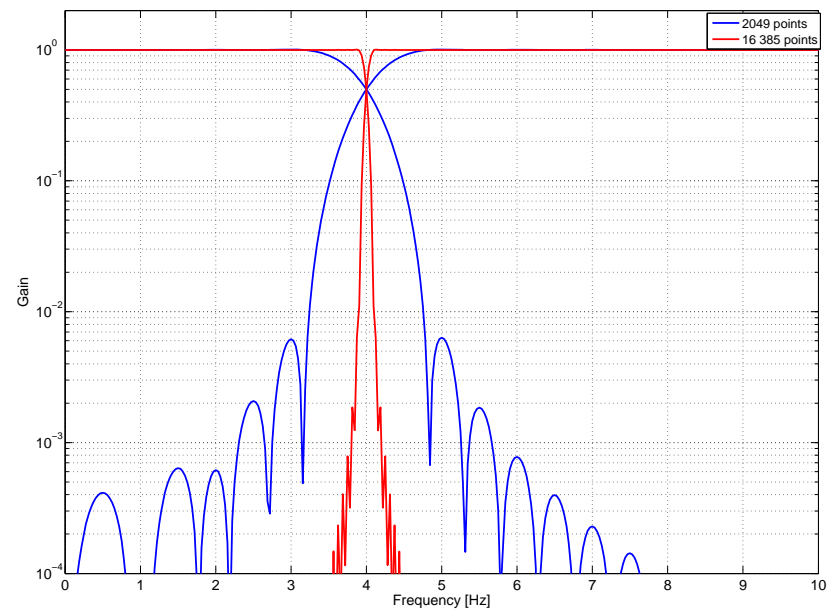

Figure 9. Frequency response of two merging filters with different filter lengths.

lays. A pure fractional time shift can be achieved by interpolation, but this would require infinite data. All realizable methods use limited time interpolation which affects gain and phase at higher frequencies.

In the time stamp update block only the time stamp is corrected by the known delays, while in the fractional delay block the fluxgate data are interpolated to the time line of the search coil data. This way all time shifts are applied with a single fractional delay filter and the influence on gain and phase is minimized. This shift was implemented as Lagrange filter and its overall spectral influence is minimal, as the higher frequency parts of the fluxgate spectrum are not used in the final merged data product.

Fluxgate data are undergoing regular in-flight calibration (Russell et al., 2014) for orthogonality, alignment, offset and gain. The required parameters for this calibration are provided by the magnetometer team and are calculated using both on-ground information as well as in-flight parameter adaptation. Furthermore, both data sets need to be transformed to a common coordinate system for merging. The chosen coordinate system for MMS is the orthogonal mounted boom system. The sequence of frequency response compensation, regular calibration and coordinate transforms is of importance. If coordinate transformation and orthogonality calibration were done first, this would result in a mix of data from different axes and therefore different frequency responses. For small rotations this error could be neglected, as in this case minor differences between these responses would be scaled by the sine of small angles. Still, for larger rotations (e.g., boom to spacecraft body coordinate system), this error is larger and frequency response compensation should be done beforehand. The best way is of course to apply frequency compensation as first step. Coordinate transforms are relying on already corrected gain and orthogonality, so those need to be done after the frequency compensation. 


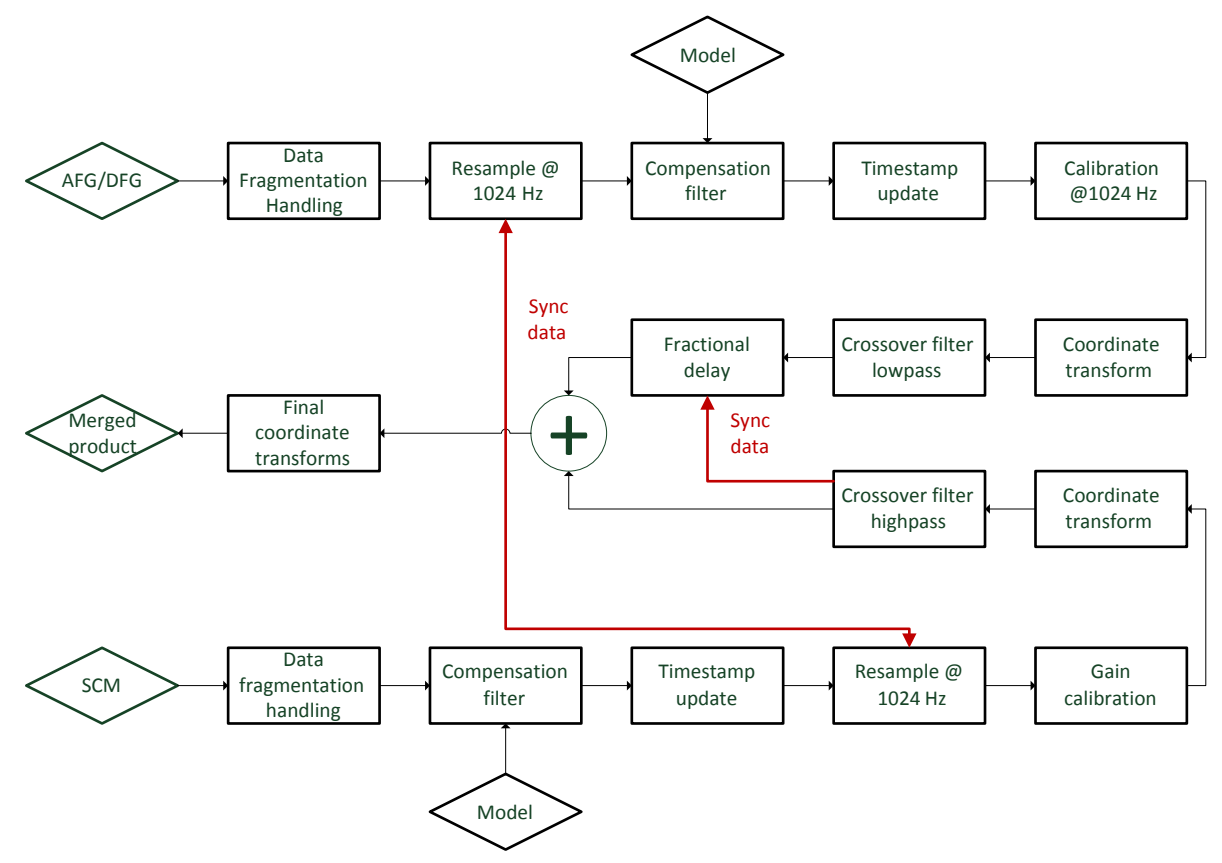

Figure 10. Data flow block diagram for merging.

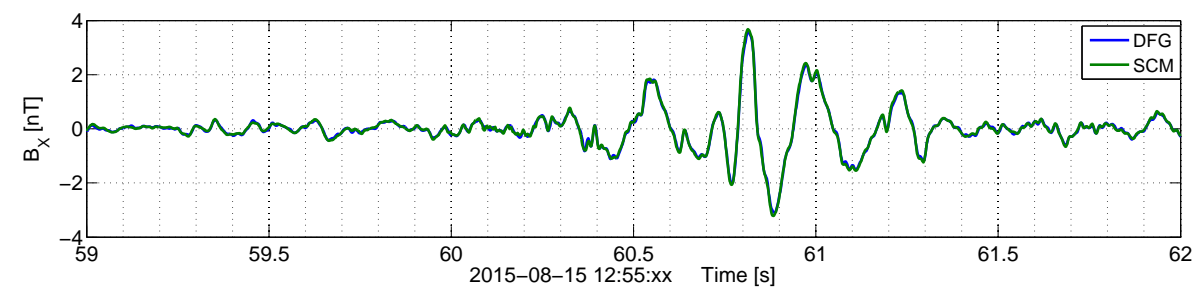

Figure 11. Comparison of MMS 3 frequency compensated data of DFG and SCM in time domain with band limitation from 4 to 64 Hz.

After merging, the data are finally transformed to the target coordinate system, i.e., geocentric solar ecliptic or magnetospheric.

\section{Evaluation}

The merged data of about 2 months were used for evaluation of the data product, as tracking of small-scale differences in time, alignment and gain required statistics on high rate burst data, which were not available at all times due to operational constraints.

A first time domain comparison (Fig. 11) in the band from 4 to $64 \mathrm{~Hz}$ shows good visual agreement between compensated data from SCM and DFG. A power spectral analysis (Fig. 12) shows the improved sensitivity of the merged product for higher frequencies. For lower frequencies the spectrum is dominated by the natural signal, as burst data are only available in regions of scientific interest that show field activity. This is in fact even the case for the higher part of the spectrum that also exceeds the noise floor of the SCM.
A more accurate analysis of the quality of the compensation models can be achieved by comparing relative gain and phase between compensated SCM and DFG data. The result of this comparison is shown in Fig. 13. The calculations for these figures were done by dividing the FFT spectra of the individual axes, which should ideally result in unity gain and zero phase for a system with perfect frequency response compensation. Data were analyzed using FFT windows with a length of 2048 points and averaging over $10 \mathrm{~min}$. Only data sets with relevant amplitudes in the frequency range 10 $64 \mathrm{~Hz}$ were taken into account, i.e., sets that have more than 10000 points above a $100 \mathrm{pT}$ magnetic field threshold with an average of at least $150 \mathrm{pT}$.

The gain plot in Fig. 13 shows a gain factor that is close to unity up to at least $30 \mathrm{~Hz}$. A clear interpretation above this frequency is difficult, as the noise increases massively. This is due to the low amplitudes of natural signals in this frequency range. These signals barely exceed the noise floor of the fluxgate, thus resulting in a poor SNR.

The phase plot does not show a significant trend but suffers from the same noise problem as the gain plot. Still the 


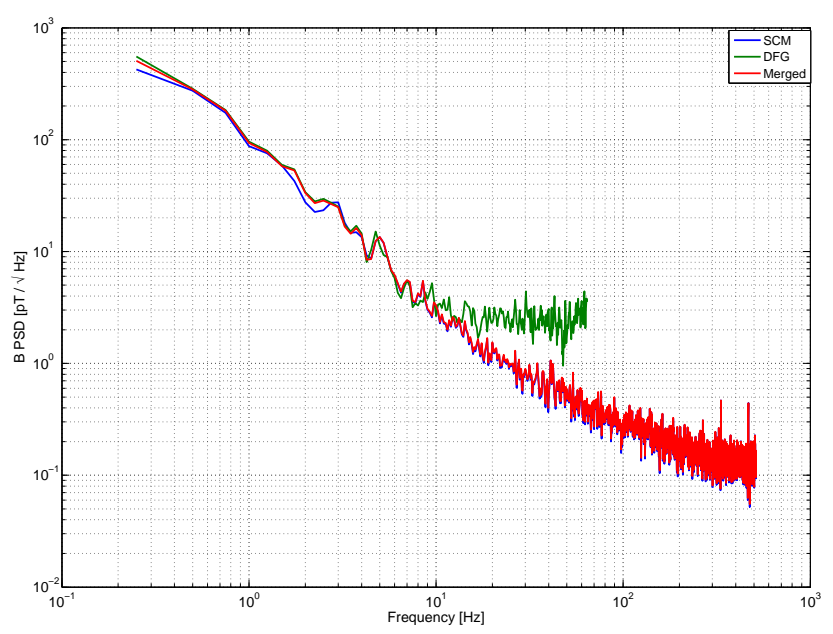

Figure 12. PSD comparison of SCM and DFG on MMS1 during a quiet field period on 20 September 2015, 06:33:31 UTC.

quality of timing determination is in this case better, as a timing error would result in a linear phase trend. For comparison purposes the time accuracy goal of $100 \mu \mathrm{s}$ was converted to linear phase trends and added to the plots. Comparing this limit to the measurement result shows no linear trends in this order of magnitude and verifies that time stamping accuracy is definitely better than $100 \mu \mathrm{s}$ in the investigated frequency band.

In addition to timing, alignment and gain were also compared by minimizing the differences between DFG and SCM measurements using linear combination of the different axes with a $3 \times 3$ alignment matrix. The result showed that the angle mismatch between DFG and SCM was in the order of 0.5 to $1^{\circ}$. This fits well to the expected differences, as SCM initial calibration assumes perfect orthogonality and alignment, while fluxgate data have full alignment and orthogonality calibration in place. The result of these comparison will be added to the SCM calibration flow in the near future.

\section{Conclusion}

The common effort of the MMS FIELDS team allowed us to create frequency response models for the FIELDS magnetic field instruments that are based on full end-to-end onground calibration. Using these models, good agreement between data from search coil and fluxgate magnetometers was achieved and data could be merged to a common product with a timing precision better than $100 \mu$ s. Furthermore, the developed methods are suitable for automated processing.

With the frequency response compensated data, alignment and gain corrections for the search coil were also generated by comparison with the in-flight calibrated fluxgate data. The resulting merged magnetometer provides a new basis for analysis of scientific events which contain frequencies ranges that are spread across two instruments.
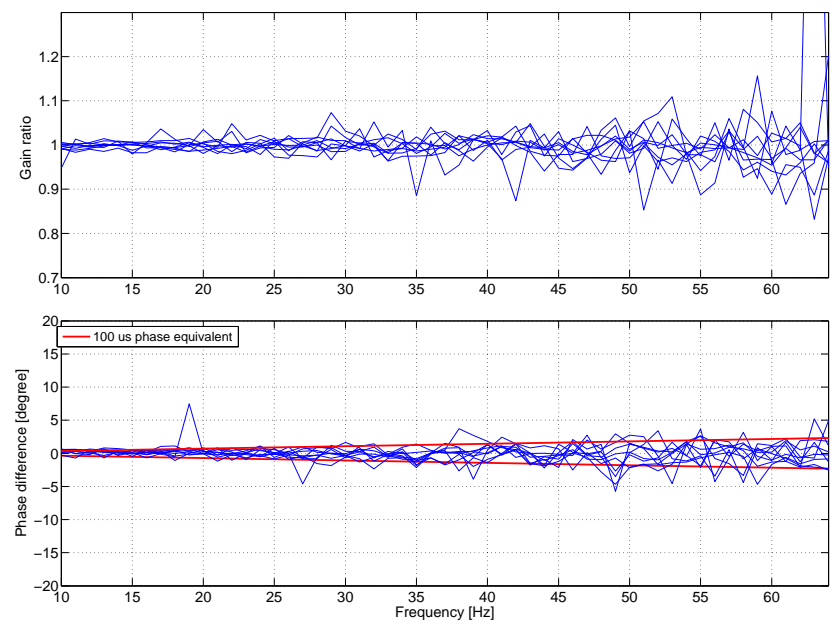

Figure 13. Comparison of gain and phase for MMS 1 SCM and DFG data.

\section{Data availability}

MMS inflight data are available at the MMS science data center at https://lasp.colorado.edu/mms/sdc/. Data are partially subject to export control or access restrictions. Access can be requested at the University of New Hampshire (ground calibration) or at the MMS Science Data Center (inflight data).

Acknowledgements. The dedication and expertise of the Magnetospheric Multiscale (MMS) development and operations teams are greatly appreciated. Work at JHU/APL, UCLA, UNH and SwRI was supported by NASA contract number NNG04EB99C. The French involvement (SCM) on MMS is supported by CNES and CNRS.

We acknowledge the use of burst L1A data from digital and analog fluxgate magnetometers and search coil magnetometers. The data are stored at the MMS Science Data Center https://lasp.colorado.edu/mms/sdc/ and are publicly available. The Austrian part of the development, operation and calibration of the DFG was financially supported by rolling grant of the Austrian Academy of Sciences and the Austrian Space Applications Programme with the contract number FFG/ASAP-844377.

\section{Edited by: V. Korepanov}

Reviewed by: two anonymous referees 


\section{References}

Alexandrova, O., Mangeney, A., Maksimovic, M., Lacombe, C., Cornilleau-Wehrlin, N., Lucek, E. A., Décréau, P. M. E., Bosqued, J.-M., Travnicek, P., and Fazakerley, A. N.: Cluster observations of finite amplitude Alfvén waves and small-scale magnetic filaments downstream of a quasi-perpendicular shock, J. Geophys. Res., 109, A05207, doi:10.1029/2003JA010056, 2004. Auster, H. U., Glassmeier, K. H., Magnes, W., Aydogar, O., Baumjohann, W., Constantinescu, D., Fischer, D., Fornacon, K. H., Georgescu, E., Harvey, P., Hillenmaier, O., Kroth, R., Ludlam, M., Narita, Y., Nakamura, R., Okrafka, K., Plaschke, F., Richter, I., Schwarzl, H., Stoll, B., Valavanoglou, A., and Wiedemann, M.: The THEMIS Fluxgate Magnetometer, Space Sci. Rev., 141, 235-264, doi:10.1007/s11214-008-9365-9, 2008.

Balogh, A., Dunlop, M. W., Cowley, S. W. H., Southwood, D. J., Thomlinson, J. G., Glassmeier, K. H., Musmann, G., Lühr, H., Buchert, S., Acuña, M. H., Fairfield, D. H., Slavin, J. A., Riedler, W., Schwingenschuh, K., and Kivelson, M. G.: The Cluster Magnetic Field Investigation, Space Sci. Rev., 79, 6591, doi:10.1023/A:1004970907748, 1997.

Burch, J. L, Moore, T. E., Torbert, R. B., and Giles, B. L.: Magnetospheric Multiscale Overview and Science Objectives, Space Sci. Rev., 199, 5-21, doi:10.1007/s11214-015-0164-9, 2015.

Cornilleau-Wehrlin, N., Chauveau, P., Louis, S., Meyer, A., Nappa, J. M., Perraut, S., Rezeau, L., Robert, P., Roux, A., De Villedary, C., De Conchy, Y., Friel, L., Harvey, C. C., Hubert, D., Lacombe, C., Manning, R., Wouters, F., Lefeuvre, F., Parrot, M., Pinçon, J. L., Poirier, B., Kofman, W., and Louarn, P.: The Cluster SpatioTemporal Analysis of Field Fluctuations (STAFF) Experiment, Space Sci. Rev., 79, 107-136, doi:10.1023/A:1004979209565, 1997.

Ergun, R. E., Tucker, S., Westfall, J., Goodrich, K. A., Malaspina, D. M., Summers, D., Wallace, J., Karlsson, M., Mack, J., Brennan, N., Pyke, B., Withnell, P., Torbert, R., Macri, J., Rau, D., Dors, I., Needell, J., Lindqvist, P.-A., Olsson, G., and Cully, C. M.: The Axial Double Probe and Fields Signal Processing for the MMS Mission, Space Sci. Rev., 199, 167-188, doi:10.1007/s11214-014-0115-x, 2016.

Haykin, S.: Adaptive Filter Theory, 4th Edn., Prentice Hall, Upper Saddle River, NJ, USA, 2002.
Le Contel, O., Leroy, P., Roux, A., Coillot, C., Alison, D., Bouabdellah, A., Mirioni, L., Meslier, L., Galic, A., Vassal, M. C., Torbert, R. B., Needell, J., Rau, D., Dors, I., Ergun, R. E., Westfall, J., Summers, D., Wallace, J., Magnes, W., Valavanoglou, A., Olsson, G., Chutter, M., Macri, J., Myers, S., Turco, S., Nolin, J., Bodet, D., Rowe, K., Tanguy, M., and de la Porte, B.: The Search Coil Magnetometer for MMS, Space Sci. Rev., 199, 257 282, doi:10.1007/s11214-014-0096-9, 2014.

Magnes, W., Pierce, D., Valavanoglou, A., Means, J., Baumjohann, W., Russell, C. T., Schwingenschuh, K., and Graber, G.: A sigma delta fluxgate magnetometer for space applications, Meas. Sci. Technol., 14, 1003-1012, doi:10.1088/0957-0233/14/7/314, 2003.

Oppenheim, A. V., Schafer, R. W., and Buck, J. R.: Discrete Time Signal Processing, 2nd Edn., Prentice Hall, Upper Saddle River, NJ, USA, 1999.

Robert, P., Cornilleau-Wehrlin, N., Piberne, R., de Conchy, Y., Lacombe, C., Bouzid, V., Grison, B., Alison, D., and Canu, P.: CLUSTER-STAFF search coil magnetometer calibration - comparisons with FGM, Geosci. Instrum. Method. Data Syst., 3, 153-177, doi:10.5194/gi-3-153-2014, 2014.

Roux, A., Le Contel, O., Coillot, C., Bouabdellah, A., de la Porte, B., Alison, D., Ruocco, S., and Vassal, M. C.: The Search Coil Magnetometer for THEMIS, Space Sci. Rev., 141, 265-275, doi:10.1007/s11214-008-9455-8, 2008.

Russell, C. T., Anderson, B. J., Baumjohann, W., Bromund, K. R., Dearborn, D., Fischer, D., Le, G., Leinweber, H. K., Leneman, D., Magnes, W., Means, J. D., Moldwin, M. B., Nakamura, R., Pierce, D., Plaschke, F., Rowe, K. M., Slavin, J. A., Strangeway, R. J., Torbert, R., Hagen, C., Jernej, I., Valavanoglou, A., and Richter, I.: The Magnetospheric Multiscale Magnetometers, Space Sci. Rev., 199, 189-256, doi:10.1007/s11214-014-0057-3, 2014.

Torbert, R. B., Russell, C. T., Magnes, W., Ergun, R. E., Lindqvist, P.-A., LeContel, O., Vaith, H., Macri, J., Myers, S., Rau, D., Needell, J., King, B., Granoff, M., Chutter, M., Dors, I., Olsson, G., Khotyaintsev, Y. V., Eriksson, C. A., Kletzing, A., Bounds, S., Anderson, B., Baumjohann, W., Steller, M., Bromund, K., Le, G., Nakamura, R., Strangeway, R. J., Leinweber, H. K., Tucker, S., Westfall, J., Fischer, D., Plaschke, F., Porter, J., and Lappalainen, K.: The FIELDS Instrument Suite on MMS: Scientific Objectives, Measurements, and Data Products, Space Sci. Rev., 199, 105-135, doi:10.1007/s11214-014-0109-8, 2014. 